\title{
USO DE CIGARROS ENTRE ADOLESCENTES DE UMA ESCOLA DE 1. GRAU DA CIDADE DE SÃO PAULO, BRASIL *
}

Glacilda Telles de Menezes Stewien ** Ruth Sandoval Marcondes **

\begin{abstract}
RSPUB9/467
STEWIEN, G. T. DE M. \& MARCONDES, R. S. Uso de cigarros entre adolescentes de uma escola de lo grau da cidade de São Paulo, Brasil. Rev. Saúde públ., S. Paulo, 13:235-43, 1979.

Resumo: Foi realizado estudo em uma escola de lo grau da rede estadual de ensino da cidade de São Paulo (Brasil), com o objetivo de investigar o habito de fumar cigarros entre adolescentes de $5^{a}$ a $8^{a}$ série, mediante a aplicação de um questionário a um total de 1.001 adolescentes. Os resultados mostraram que o hábito de fumar cigarros $\dot{e}$ bastante difundido entre os adolescentes, aumentando proporcionalmente da $5^{a}$ para a $8{ }^{a}$ série. $O$ maior aumento na proporção de fumantes foi verificado da $6^{a}$ para a $7^{a}$ série ( $8,3 \%$ para $21,5 \%$ ). Observou-se que o hábito de fumar dos adolescentes atingiu, no grupo etário de 16 anos, proporçōes semelhantes às dos adultos, e que o hábito de fumar cigarros levou, em média, aproximadamente dois anos para se estabelecer entre os adolescentes investigados.
\end{abstract}

UNITERMOS: Fumo. Estudantes.

I NTRODUCAO

Em estudo patrocinado pela Organização Panamericana da Saúde (OPAS), Joly 8 (1975) mostrou a alta prevalência do hábito de fumar cigarros na população urbana de oito capitais da América Latina. Nesta investigação foi possível verificar que a percentagem de fumantes do sexo masculino chegou a sobrepujar a dos Estados Unidos em seis das oito cidades estudadas, incluindo a capital de São Paulo, que acusou a cifra de $54 \%$ de fumantes na faixa etária de 15 a 74 anos.
É fato reconhecido que sérias doenças decorrem do uso de cigarros, devendo-se destacar o câncer da cavidade oral, da laringe, do esôfago, da faringe e do pulmão $1,2, \hat{0}, \mathbf{6}, \hat{\imath}, 10,11$. Estudo realizado em 10 grandes centros urbanos da América Latina ${ }^{9}$ mostrou que já durante o período de 1962 a 1964 as doenças relacionadas com o uso de cigarros foram responsáveis por nada menos do que $20 \%$ das mortes no grupo etário masculino de 35 a 64 anos. Com o crescente consumo de cigarros nos

* Parte da Dissertação de Mestrado apresentada à Faculdade de Saúde Pública da USP, em 1977.

* Do Departamento de Prática de Saúde Pública da Faculdade de Saúde Pública da USP Av. Dr. Arnaldo, 715 - 01255 - São Paulo, SP - Brasil. 
STEWIEN, G. T. de M. \& MARCONDES, R. S. Uso de cigarros entre adolescentes de uma escola de 1" grau da cidade de São Paulo. Brasil. Rer. Saúde públ., S. Paulo, 13: 235-43, 1979.

dias de hoje é bem possivel que esta cifra esteja aumentando.

Sendo, pois, o problema do uso de cigarros de elevada expressão entre nós, resolveu-se estudá-lo detalhadamente entre adolescentes da cidade de São Paulo.

O presente trabalho apresenta a prevalência e a distribuição do hábito de fumar cigarros entre adolescentes de ambos os sexos de uma Escola de 1? Grau da rede pública estadual da cidade de São Paulo, incluindo alunos da $5^{a}$ à $8^{a}$ série.

\section{MATERIAL E MÉTODOS}

População estudada: A Escola onde foi realizada a investigação recebe alunos de várias classes sociais, sendo que grande parte pertence à classe média.

Foram investigados os alunos da $5^{a}$ série (a maioria com 11 anos de idade), da $6^{\prime \prime}$ série (a maioria com 12 anos), da $7^{*}$ série (a maioria com 13 anos) e da $8^{4}$ série (a maioria com 14 anos)*. Em 1975, ano da realização da pesquisa, o número total de alunos matriculados nas diferentes classes da $5^{\text {a }}$ à $8^{a}$ série era de 1.102 (567 do sexo masculino e 535 do sexo feminino), dos quais 1.001 (523 do sexo masculino e 478 do sexo feminino) estiveram presentes na ocasião do inquérito, o que representa mais de $90 \%$ do universo total.

Coleta de dados: As informações sobre o hábito de fumar foram obtidas por meio de um questionário preenchido simultaneamente e de modo anônimo pelos alunos em suas respectivas classes. Todos os questionários foram aplicados em um único dia por docentes e alunos da Faculdade de Saúde Pública/USP e por alguns professores da Escola.

Em sua forma original o questionário constava de questões abertas; após o pré-teste, realizado em escola cujos alunos eram de nivel sócio-econômico semelhante aos da investigação, foi elaborado o questionário definitivo, que passou a conter trinta e quatro questões abertas e fechadas, dividido em três partes. A primeira parte, contendo catorze perguntas ( 1 a 14), visava a obter informaçñes de caráter geral do aluno e classificá-lo de acordo con sua condição de fumante ou não fumante, segundo critério apresentado a seguir. A segunda parte (perguntas 15 a 20) deveria ser respondida apenas pelos adolescentes que não fumavam ou que haviam deixado de fumar antes da pesquisa. A terceira parte (perguntas 21 a 34 ) deveria ser preenchida pelos alunos que fumavam no momento da realização da pesquisa $: 2$.

Classificação: Os adolescentes foram classificados em dois grupos:

1. Fumantes, subdivididos em:

a. Fumante regular - o adolescente que, por ocasião da pesquisa, fumava cigarros todos os dias;

b. Fumante ocasional - 0 adolescente que, por ocasião da pesquisa, fumava esporadicamente.

2. Não fumantes, subdivididos em.

a. Ex-fumante regular - o adolescente que informou ter fumado regularmente no passado;

b. Ex-fumante ocasional -o adolescente que informou ter fumado ocasionalmente no passado;

c. Nunca-fumante - 0 adolescente que jamais fumou.

\section{RESULTADOS}

1. Hábito de fumar cigarros segundo a série escolar.

Na Tabela 1 são apresentados os resultados sobre a prevalència do hábito de fumar cigarros entre os adolescentes inves-

* A idade típica dos alunos pertencentes a una série escolar corresponde a idade da maioma deles naquela série. 


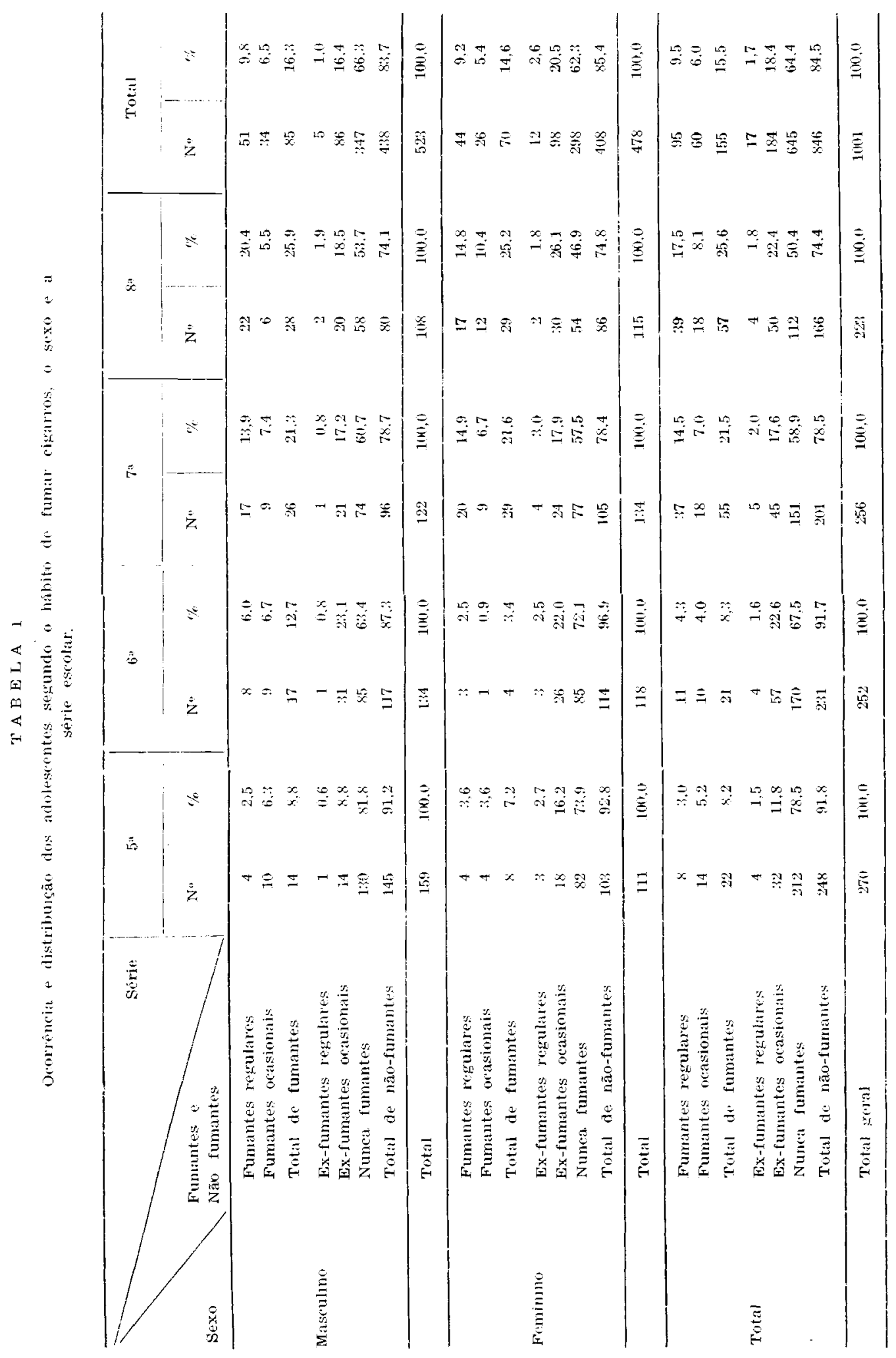


STEWIEN, G. T. de M. \& MARCONDES, R. S. Uso de cigarros entre adolescentes de uma escola de $1^{\circ}$ grau da cidade de Săo Paulo, Brasil. Rev, Saúde públ., S. Paulo, 13: 285-43, 1979.

tigados, de acordo com o critério de classificação estabelecido. Verifica-se, inicialmente, que a proporção de fumantes regulares e ocasionais aumenta progressivamente de $8,2 \%$, na $5^{\text {a }}$ série, para $25,6 \%$, na 8 e série. $O$ maior aumento, nesta proporção, verificou-se da $6^{\text {a }}$ para a $7^{\text {a }}$ série, quando passou de $8,3 \%$ para $21,5 \%$.

No sexo masculino, a proporção de fumantes regulares aumenta da 5 a para a $8^{\text {a }}$ série $(2,5 \%$ para $20,4 \%)$, permanecendo a proporção de fumantes ocasionais praticamente inalterada nas quatro séries investigadas. No sexo feminino, ambas as proporções aumentam da $5^{\mathrm{a}}$ para a $8^{a}$ série, como se vê ainda na Tabela 1. Isto mostra que parte considerável das adolescentes fuma cigarros em base ainda experimental durante este período escolar. Já no sexo masculino, o hábito de fumar cigarros se torna regular para a maioria dos adolescentes a partir da $7^{\text {a }}$ série do 10 Grall.

Outra observação que merece ser destacada é a que se refere à proporção dos adolescentes nunca fumantes, que vai diminuindo gradativamente, em ambos os sexos, através das quatro últimas séries do 19 Grau. A queda maior foi observada da $5^{a}$ $(78,5 \%)$ para a $6^{\text {a }}$ série $(50,4 \%)$. Ai também verificamos que a proporção de ex-fumantes ocasionais aumenta em dobro, isto é, de $11,8 \%$ para $22,6 \%$.

\section{Hábito de fumar cigarros segundo a idade e sexo}

A Figura 1 mostra a proporção de tulmantes regulares e ocasionais segundo a idade e o sexo. Verifica-se que o hábito de fumar cigarros dos adolescentes aumenta progressivamente com a idade, atingindo cifras relativamente elevadas aos 16 anos de idade: $53,3 \%$ para o sexo masculino e $46,6 \%$ para o feminino.

Na Figura 2 os escolares foram classificados em 3 grupos de acordo com a idade relacionada à série escolar em que se encontravam. Segundo esta classificação, verifica-se que a grande maioria dos fumantes de ambos os sexos tinha idade superior à tipica da série que freqüentavam. Isto mostra que são os alunos mais velhos da classe que mais fumam nas quatro séries estudadas.

\section{Consumo de cigarros}

A quantidade de cigarros consumida pelos adolescentes de ambos os sexos é apresentada na Tabela 2 . Verifica-se que o consumo de cigarros aumenta progressivamente da 5. para a $8^{9}$ série entre os fumantes do sexo masculino. Dentre os fumantes da $8^{\text {a }}$

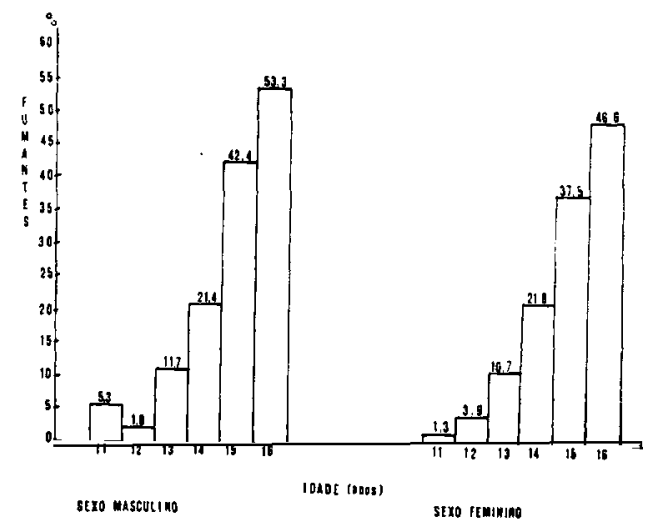

Fig. 1 - Distribuição de fumantes, segundo a idacte o sexo.

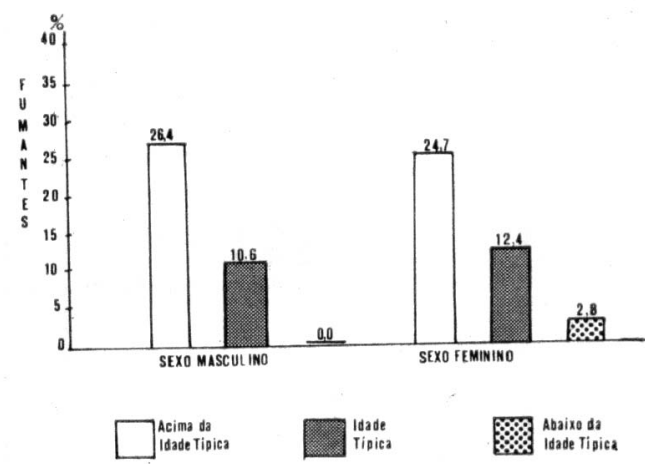

Fig. 2 - Distribuiçăo de fumantes de acordo com a idade e a sexo relacionada à série escolar. 


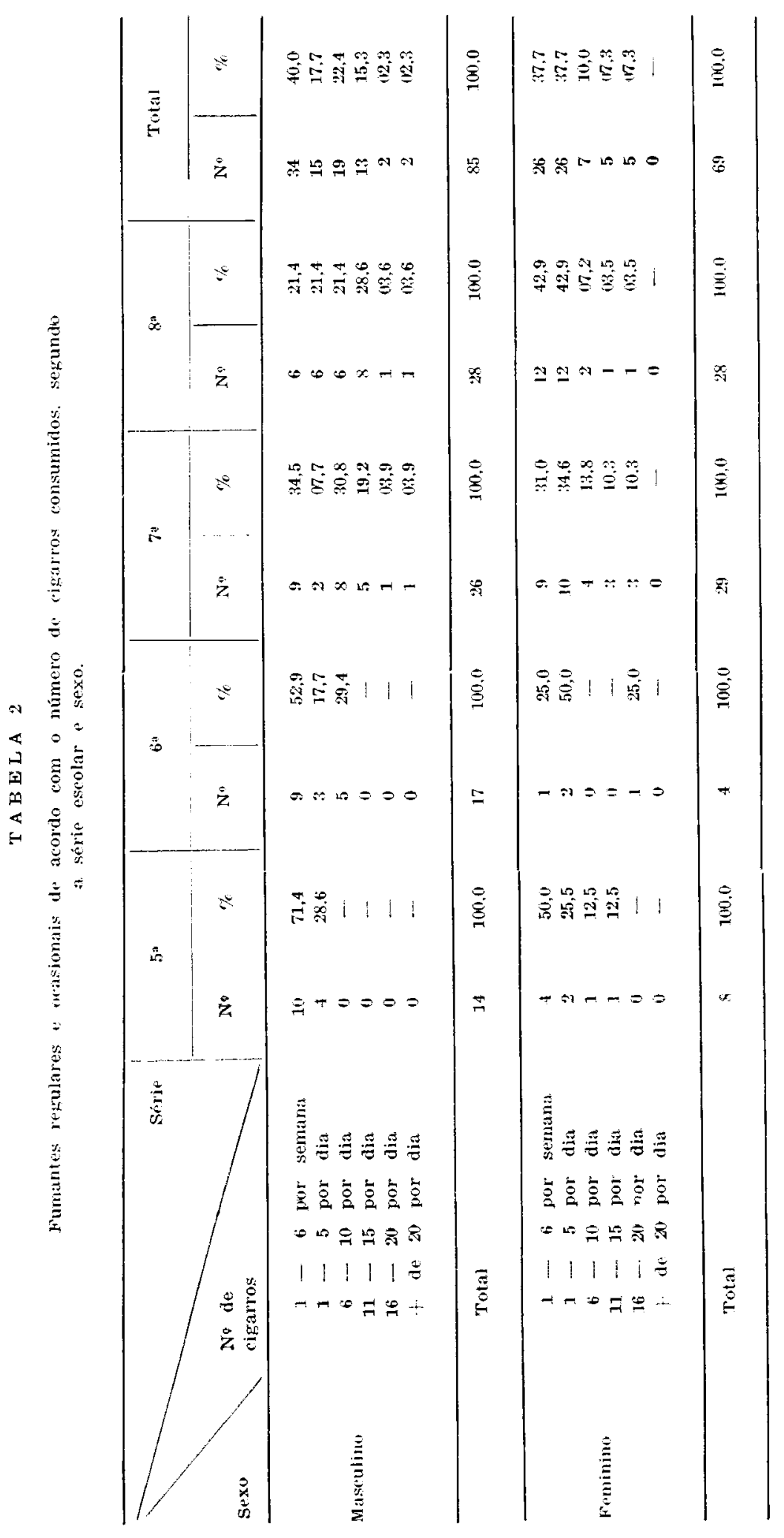


STEWIEN, G. T. de M. \& MARCONDES, R. S. Uso de cigarros entre adolescentes de uma escola de 10 grau da cidade de São Paulo, Brasil. Rev. Saúde públ., S. Paulo, 13: 235-43, 1979.

série, nada menos do que $35,8 \%(28,6 \%+$ $3,6 \%+3,6 \%)$ consomem diariamente meio maço de cigarros (11 ou mais cigarros). Para o sexo feminino, o consumo de cigarros acusou valores menores, o que concorda com a observação anteriormente feita de que as adolescentes estudadas ainda fumam, em sua maioria, em base experimental.

$\mathrm{Na}$ Tabela 3 estão classificados os fumantes regulares e ocasionais de acordo com o tipo de cigarro consumido. Observase que a grande maioria dos adolescentes de ambos os sexos (mais de $90 \%$ ) prefere fumar cigarros com filtro. É importante também ressaltar o fato de que todos os que fumavam cigarros de qualquer tipo eram fumantes que consumiam mais de 15 cigarros por dia.

\section{Estabelecimento do hábito de fumar}

Os fumantes regulares e ocasionais foram inquiridos sobre a idade em que fumaram pela primeira vez. De acordo com as respostas fornecidas, os adolescentes de ambos os sexos foram distribuidos em quatro grupos etários. Conforme mostra a Tabela 4, aproximadamente $70 \%$ $(34,8 \%+34,0 \%)$ dos fumantes começaram a fumar quando tinham idade de 10 a 14 anos incompletos.

Segundo as respostas fornecidas pelos fumantes regulares sobre quando começaram a fumar regularmente, os adolescentes foram, novamente, distribuidos em quatro grupos etários. A Tabela 5 mostra que $70 \%(38,9 \%+31,1 \%)$ dos fumantes começaram a fumar regularmente quando tinham 12 a 16 anos incompletos. De acordo com as informações prestadas pelos alunos fumantes regulares, referentes à idade em que fumaram pela primeira vez $e^{\cdot}$ à idade em que se tornaram fumantes regulares, verificamos que $o$ hábito de fumar levou, em média, aproximadamente 2 anos para se estabelecer entre os adolescentes de ambos os sexos.

T A B E L A ?

Distribuição dos fumantes segundo sexo e tipo de cigarros.

\begin{tabular}{l|l|l|l|}
\hline Sexo \\
de cigarro
\end{tabular}

$\begin{array}{lcccr}\text { Sem filtro } & 3 & 3,5 & 1 & 1,4 \\ \text { Com filtro } & 78 & 91.8 & 67 & 95.7 \\ & & & & \\ \text { Qualquer tipo } & 4 & 4,7 & 2 & 2.9\end{array}$

\begin{tabular}{ccccc}
\hline Total & 85 & 100,0 & 70 & 100,0 \\
\hline
\end{tabular}


STEWIEN, G. T. de M. \& MARCONDES, R. S. Uso de cigarros entre adolescentes de uma escola de 1o grau da cidade de São Paulo. Brasil. Rev. Saúde públ., S. Paulo, 13: $235-43,1979$.

T A B E LA 4

Idade em que os adolescentes fumantes atuais fumaram pela primeira rez, segundo o sexo.

\begin{tabular}{|c|c|c|c|c|c|c|c|c|c|}
\hline \multirow[b]{2}{*}{ Idade (anos) } & & & \multirow[t]{2}{*}{ Sexo } & \multicolumn{2}{|c|}{ Masculino } & \multicolumn{2}{|c|}{ Feminino } & \multicolumn{2}{|c|}{ Total } \\
\hline & & & & No & $\%$ & No & $\%$ & N" & $\%$ \\
\hline 8 & 1 & 10 & & 11 & 14,1 & 8 & 12,7 & 19 & 13.5 \\
\hline 10 & 1 & 12 & & 26 & 33,3 & 23 & 36,5 & 49 & 34,8 \\
\hline 12 & $1-$ & 14 & & 24 & 30,8 & 24 & 38,1 & 48 & 34,0 \\
\hline 14 & $\mathrm{e}$ & + & & 17 & 21,8 & 8 & 12.7 & 25 & 17,7 \\
\hline
\end{tabular}

14 alunos fumantes deixaram de responder.

T A B E L A 5

Idade em que os adolescentes fumantes regulares começaram a fumar regularmente.

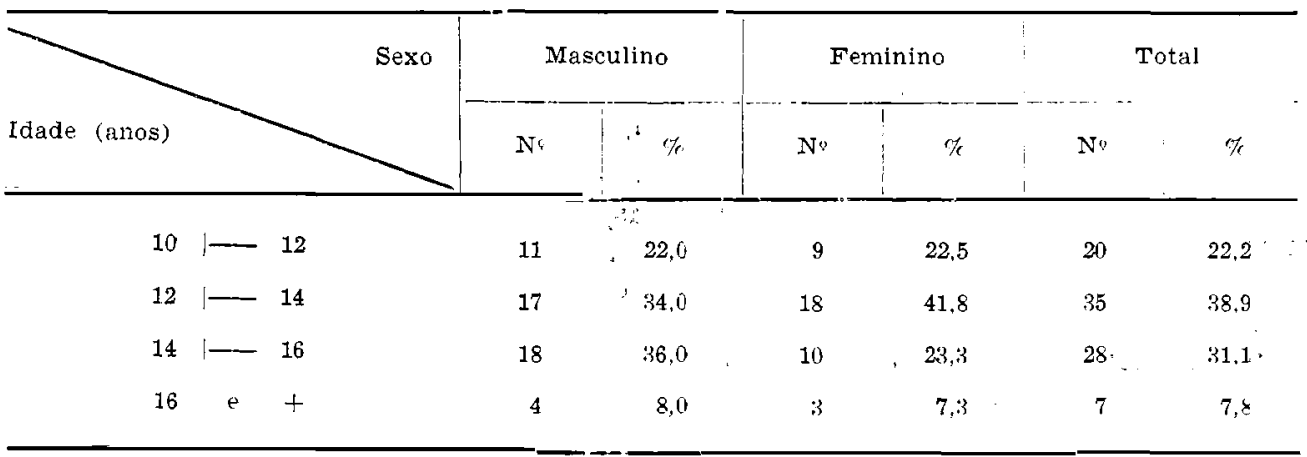

5 alunos deixaram de responder.

\section{DISCUSSĀO}

Os resultados mostram que o hábito de fumar cigarros é bastante difundido entre os adolescentes investigados, aumentando progressivamente da $5^{a}$ para a $8^{a}$ série do 10 Grau. Nesta última série escolar vimos que cerca de $25,0 \%$ dos adolescentes fumam. Observamos também que a proporção de fumantes aumenta mais da $6^{\mathrm{a}}$ para a $7^{\text {a }}$ série. Por outro lado, verificamos que a percentagem dos nunca fumantes diminui gradualmente para ambos os sexos, tendo sido registrada a maior queda da $5^{\mathrm{a}}$ para a 6a série. Aí também observamos que a proporção dos ex-fumantes ocasionais aumenta cerca de duas vezes. Estes resultados, sem dúvida, marcam a $6^{\text {a }}$ série como o momento critico em que a maioria dos adolescentes decide se vai fumar no futuro ou não. Este fato é de extrema importância e deve ser considerado na aplicação de programas anti-fumo na escola.

Estudos realizados nos Estados Unidos por uma equipe multiprofissional da Universidade de Illinois * acusaram em 1967 o mesmo resultado, com a única diferença de que o momento crítico se mostrou não na 6a, mas na. $8^{\text {a }}$ série. Esta comparação indica uma tendência segundo a qual, os adolescentes por nós estudados se decidem mais cedo a fumar do que os seus colegas norte-americànos. 
STEWIEN, G. T. de M. \& MARCONDES, R. S. Uso de cigarros entre adolescentes de uma escola de 1o grau da cidade de São Paulo, Brasil. Rev. Saúde públ., S. Paulo, 13: $235-43,1979$.

Os resultados apresentados na Fig. 1 mostram que o hábito de fumar cigarros alcança, no grupo etário de 16 anos, proporções semelhantes aos dos adultos. $O$ inquérito realizado por Joly 8 (1975) revelou que, da população masculina da cidade de São Paulo na faixa etária de 15 a 24 anos, 40,0\% fumam. Para o sexo feminino esta proporção foi de $16,0 \%$, portanto muito inferior àquela por nós encontrada para os grupos etários de 15 e 16 anos. As percentagens do presente estudo em muito se assemelham às de um inquérito realizado na Finlândia ${ }^{10}$, em nivel nacional, que acusou para os grupos etários de 12 , $13,14,15$ e 16 anos, respectivamente, $6,5 \%$, $10,9 \%, 20,6 \%, 32,6 \%$ e $48,0 \%$ na população masculina. $\mathrm{Na}$ população feminina, foram encontradas percentagens aproximadamente iguais. Assim, a diferença marcante que antes existia entre o número de fumantes de ambos os sexos tende a desaparecer nos dias atuais. $O$ mesmo fato venı sendo observado em diversos paises, como por exemplo nos Estados Unidos, Suécia e Dinamarca ${ }^{10} . \quad \mathrm{Na}$ atualidade muito mais mulheres fumam do que antigamente, mostrando a grande popularidade que o hábito de fumar goza nos dias de hoje.

A generalização do uso de cigarros pode ser atribuida à propaganda em prol do cigarro que, através de mensagens sedutoras diariamente dirigidas ao grande público, consegue atrair cada vez mais os jovens. É importante saber que a referida propaganda já é proibida em alguns paises como os Estados Unidos, a Suécia e a Itália. Tal providência deveria ser adotada também no Brasil.

A Tabela 2 demonstra o número apreciável de cigarros consumidos pelos adolescentes, já existindo grandes fumantes no sexo masculino, que chegam a fumar diariamente um maço de cigarros ou mais. É fato bem conhecido a existência entre os fumantes de uma tendência geral no sentido de aumentar o consumo de cigarros à me- dida que o hábito de fumar evolui. Deste modo, é provável que muitos dos jovens investigados se tornarão fumantes inveterados no futuro, consumindo uma média diária de dois maços de cigarros ou mais.

Conforme mostra o presente estudo, a grande maioria dos jovens fumantes de ambos os sexos prefere fumar cigarros com filtro. Esta tendência generalizada deve ser atribuída também à propaganda a favor de tal tipo de cigarros, do que propriamente à preferência real dos consumidores. Esta observação mostra, mais uma vez, a poderosa influência que a publicidade do cigarro tem sobre nossa população adolescente.

Finalmente, o estudo também revelou que o hábito de fumar se inicia cedo entre os adolescentes, estabelecendo-se, em ambos os sexos, dentro de um prazo aproximado de dois anos. Observação semelhante foi feita por Colli 3 (1972) em inquérito realizado na cidade de São Paulo sobre aspectos globais de saúde do adolescente. Isto mostra que programas anti-fumo devem ser iniciados já na $5^{\text {a }}$ série do $1^{\circ}$ grau. com vista à prevenção do uso de cigarros entre adolescentes.

\section{CONCLUSOES}

As principais conclusões deste trabalho são:

1. O número de adolescentes que fumam atinge na $8^{\text {a }}$ série do 10 Grau a percentagem de $25,6 \%$;

2. A 6a série do 10 Grau foi identificada como sendo o período crítico no qual 0 aluno decide se vai se tornar fumante regular ou não. Conseüentemente, programas anti-fumo devem ser iniciados na $5^{\text {a }}$ série do 19 Grau, a fim de prevenir o hábito de fumar cigarros entre os adolescentes;

3. O consumo de pelo menos meio maço de cigarros por dia por $19,9 \%$ dos fumantes do sexo masculino e $14,6 \%$ do sexo feminino mostra a 
STEWIEN, G. T. de M, \& MARCONDES, R. S. Uso de cigarros entre adolescentes de uma escola de 10 grau da cidade de São Paulo, Brasil. Rev. Saúde públ., S. Paulo, 13: 235-43, 1979.

gravidade do problemla na população estudada;

4. A maioria dos fumantes se situa entre os alunos mais velhos das respectivas classes $(26,4 \%$ do sexo masculino e $24,7 \%$ do sexo feminino).

5. O hábito de fumar levou cerca de dois anos para se estabelecer entre os adolescentes invesigados.

RSPUB9 $/ 467$

STEWIEN, G. T. de M. \& MARCONDES, R. S. /Smoking habits among students of a public school of the city of S. Paulo, Brazil./ Rev. Saúde públ., S. Paulo, $13: 235-43,1979$.

ABSTRACT: A study was carried out at a public school of the city of Saio Paulo to investigate the cigarette smoking habits of 5th-to 8th-graders in 1975. Over 90 per cent of 1002 students answered a questionnaire on their smoking habits. The percentage of regular and occasional smokers increased from 8 per cent in the 5th grade to 17.5 per cent in the 8th grade. The greatest increase occurred from the 6th to the 7 th grade. There was a real difference in the percentage of smokers between the boys and girls, particularly in the 8 th grade when 36 per cent of the boys smoked half a pack of cigarettes or more per day. Boys and girls who smoked reported an interval of about two years between their first cigarette and becoming regular smokers. The paper concludes that anti-smoking programs should start in school at the 5th grade.

UNITERMS: Smoking. Students.

\section{REFERENCIAS BIBLIOGRAFICAS}

1. ADVISORY COMMITTEE TO THE SURGEON GENERAL. The health consequences of smoking. Washington, D. C., Public Health Service, 1971. (PHS Publ. 7513).

2. BALDWIN, B. H. et al. Teaching about smoking and health. Chicago, Ill., Office of the Superintendent of Public Instruction and Department of Public Health, 1964.

3. COLLI, A. S. Adolescentes: aspectos globais de saúde. São Paulo, 1972. [Tese de Doutoramento - Faculdade de Medicina da USP]

4. CRESWELL, W. $H$. et al. University of Illinois antismoking education study. Illinois $J$. Educ., 60(30):27-37, 1969.

5. EL HABITO de fumar $y$ las enfermedades: nueva exposición de los hechos. Cron. Org. mund Salud, 29:434-41. 1975.

6. HAMMOND, E. C. The effects of smoking. Sci. amer., 207(7):3-15, 1962.

7. HEALTH consequences of smoking: 1969 supplement, of the 1967 Public Health Service Review. Washington, D. C.
Public Health Service, 1969, (PHS Publ. 1696-2).

8. JOLY, D, J, El habito de fumar cigalrillos en America Latina: una encuesta en ocho ciudades. Bol. Ofic, santit. panamer, 79:93-110, 1975.

9. PUFFER, R. R. \& GRIFFITH, G. W. Caracteristicas de la mortalidad urbana: informe de la Investigación Interamericana de Mortalidad. Washington, D. C., Organización Panamericana de la Salud, 1968. (OPAS - Publ. cient., 151).

10. RAMSTRöM, I. M. et al. A National Smoking Control Programme. Stockholm, National Smoking and Fealth Association, NTS, 1973.

11. SALBER, E. J. Facts about smoking and health. Chicago, Science Research Associates, 1968.

12. STEWIEN, G. T. de M. $O$ adolescente $e$ o fumo. São Paulo, 1977. [Dissertação de Mestrado - Faculdade de Saúde Pública da USP]

Recebido para publicacão em 16/08/1979 Aprovado para publicação em 22/03/1979 